\title{
Paediatric Tuberculosis - Can we eradicate it?
}

\section{Nibedita Rath*}

Open Source Pharma Foundation, Bangalore-560012, Karnataka, India

*Corresponding author: Nibedita Rath, Open Source Pharma Foundation, World Trade Centre, 22nd floor, \#N 2201, 26/1 Dr Rajkumar Road, 26/1 Dr Rajkumar Road, Bangalore, 560055, Karnataka, India, Tel:+91.8067935668;E-mail: nibi2009@gmail.com

Received date: April 30, 2018; Accepted date: May 16, 2018; Published date: May 23, 2018

Copyright: @ 2018 Rath N. This is an open-access article distributed under the terms of the Creative Commons Attribution License, which permits unrestricted use, distribution, and reproduction in any medium, provided the original author and source are credited.

\begin{abstract}
Tuberculosis is on the rise and remains a problem of enormous magnitude regardless of the existence of discrete measurements. It is re-emerging and there is a global threat due to the increasing number of cases among children and adults. Studies have shown that children contribute significantly to the disease burden and the morbidity and mortality, associated with the disease is grievous, particularly in endemic areas. Children are more prone to develop a life-threatening form of tuberculosis due to an immature immune system. Paediatric infections are a repository, from which probability of the evolution of new adult case is high.
\end{abstract}

\section{Key words:}

Paediatric Tuberculosis; Drug resistance; Pharmacokinetics (PK); Diagnosis; Drug regimen; Immune system; Metformin

Paediatric TB has been relatively pretermitted in comparison to adult TB. The diagnosis of paediatric tuberculosis is difficult and hence the true picture of drug-sensitive and drug-resistant TB in children is unknown. In 2013, 0.55 million children have been estimated to develop TB $[1,2]$ as shown by the World Health Organization (WHO), which is contradicted to the recent study, that the number of children that develop TB has touched 1 million, which is way above the estimation made by WHO. This population is neglected due to the perception that $\mathrm{TB}$ does not get transmitted by children. Of late the inclusion of children is happening notably in global and national TB agendas. Tuberculosis is on the rise in the 3rd world countries, regardless of the existence of discrete measurements. It is re-emerging and there is a global threat due to the increasing number of cases among children and adults. Even though the number of cases of children acquiring TB is less in comparison to the adult, still then paediatric infections are a repository from which probability of the evolution of new adult case is high.

Children are associated with wide range of tuberculosis, which ranges from pulmonary $\mathrm{TB}$ to extra pulmonary $\mathrm{TB}$ (mainly $\mathrm{TB}$ Meningitis) [3]. The duration of treatment and drug doses differ by disease extent and location. Determination of Pharmacokinetics (PK) value is critical and should equate with the efficacy of paediatric TB regimen, as this piece of information is necessary for dose optimization and which can also be utilized in shortening the treatment duration.

Children acquire $\mathrm{TB}$ from adults and they represent a large percentage of a pool from which further TB cases arise. Infant and children have $8-10 \%$ lifetime risk of developing tuberculosis from TB infection $v s 5 \%$ if infection acquired as an adult. Tuberculosis diagnosis [4] in children is very difficult particularly in infants and small children due to the insufficient specimen and fewer TB bacteria in the sputum. Paediatric diagnosis is subsequent to a new discovery of an adult case and based on TB skin test, chest X-ray and followed by physical symptoms. Interpretation of these diagnostic outputs is sometimes difficult and inconclusive. In spite of the emergence of new diagnostic techniques, it has not shown any satisfactory outcome in paediatric diagnosis. Improved techniques need to be in place for early diagnosis of paediatric tuberculosis. Recently a team of investigators from the University of Cape Town (UCT) have shown that using oral swabs, testing samples from the tongue, cheeks and gums of patients can detect TB in both children and adults. The sensitivity rate of 49 samples that have been collected is quite promising, which is about $92 \%$. The researchers have found that scrapped tissue from the tongue yields the best result. Further investigation is in progress before it rolls out for its full fledge use [5].

The tuberculosis regimens in children are same as that of the adult except for the facts that the doses are different $[6,7]$. Pharmacokinetics and toxicity profiling is must in order to estimate the right dosing and to maintain safety and enhance tolerability. As of now potency studies for paediatric tuberculosis have not been gained much attention. There is an immediate requirement to focus on research to truncate the treatment duration for paediatric patients with less severe drug resistance TB. Discovery of new drug regimen with less toxicity profile for severe paediatric TB should be prioritized.

Advanced diagnostic techniques should be used for effective screening of paediatric tuberculosis. The standard screening methods which consist of tuberculin test, variation in chest X-ray and mainly rely on documented exposure to an infection [8] are not sufficient enough. Better and improved diagnostic methodologies should be in place for precise detection of paediatric TB. The $1^{\text {st }}$ and $2^{\text {nd }}$ line TB regimen with improvised formulations are needed to nullify death rate among children due to tuberculosis. The clinical representation of TB in adult and paediatric patients is not same. This basic difference must be taken into account before executing new tests on children with TB.

The lack of proper understanding of the complex interaction between pathogen and its host (human) has been an important factor hindering the development of new drugs for tuberculosis. Pharmaceutical industries are not willing to invest in developing and marketing products that are intended for $3^{\text {rd }}$ world diseases area, as such products will not be profitable for the companies. 
Few things that need immediate attention to curb the issue are listed below:

An immediate need to shorten the treatment duration for less severe drug resistance paediatric TB.

Evaluation of current regimen for more severe drug resistance paediatric TB.

Determination of pharmacokinetic parameters based on severity and location of the disease.

Dose adjustment based on age and weight in infant and children less than 5 yrs.

Optimization of first-line antituberculosis drugs for children under 5 yrs.

Research findings [9] have suggested that the widely used metformin, an antidiabetic drug has been shown to be efficacious against tuberculosis. Metformin works by modulating the immune response by augmenting macrophage function by AMPK activation and promotion of mitochondrial ROS that kills TB.

Metformin should be studied in the paediatric clinical trial set up and implemented as an adjunct therapy in paediatric tuberculosis if found efficacious.

\section{References}

1. World Health Organization(2014) Tuberculosis Fact Sheet

2. Dodd PJ, Sismanidis C, Seddon JA (2016) Global burden of drug-resistant tuberculosis in children: a mathematical modelling study. Lancet Infect Dis 16: 1193-1201.

3. Esposito S, Tagliabue C, Bosis S (2013) Tuberculosis in Children. Mediterr J Hematol Infect Dis 5: 2013064.

4. Jain P, Jain I (2014) Oral Manifestation of Tuberculosis: Step towards Early Diagnosis. J Clin Diagn Res 8: 12.

5. Wood RC, Luabeya AK, Weigel KM, Wilbur AK, Jones-Engel L, et al (2015) Detection of Mycobacterium tuberculosis DNA on the oral mucosa of tuberculosis patients. Sci Rep 5: 8668.

6. Kendall EA (2017) Tuberculosis in Children: under-counted and undertreated. Lancet Glob Health 5: e845 to e846.

7. TB drugs for Children (2013) Treatment Action Group.

8. Roya-Pabon CL, Perez-Velez CM (2016) Tuberculosis exposure, infection and disease in children: a systematic diagnostic approach. Pneumonia 8: 23.

9. Singhal A, Jie L, Kumar P, Hong GS, Leow MK, et al (2014) Metformin as adjunct antituberculosis therapy. Sci Transl Med 6: 263ra159. 